\title{
A influência da variação da curvatura da base do braquete em uma união ortodôntica submetida a diferentes cargas, através do método dos elementos finitos*
}

\author{
Cláudio Pereira Viana**, Ênio Tonani Mazzieiro ${ }^{\star \star \star}$, Janes Landre Júnior ${ }^{\star \star \star \star ~}$
}

\begin{abstract}
Resumo
Este trabalho procurou avaliar, através do Método dos Elementos Finitos, a resistência ao deslocamento de quatro diferentes bases de braquetes "straight-wire" (Morelli, Unitek, A-Company e Abzil-Lancer) na aplicação de forças de torção e cisalhamento, correlacionando essa resistência à melhor adaptação das bases à superfície vestibular dentária. As curvaturas das bases de braquetes utilizados no presente estudo foram descritas em um estudo prévio, enquanto o contorno dentário foi obtido a partir do escaneamento e cálculo da curvatura média vestibular do canino inferior de uma amostra de 30 indivíduos brasileiros. Empregou-se a análise tridimensional por Elementos Finitos da interface esmalte vestibular/cimento/base do braquete para cada marca testada. Forças de $1 \mathrm{~N}$, promovendo deslocamento lateral e torção, foram aplicadas no centro da base do braquete e observou-se a distribuição desses esforços sobre os modelos assim como as tensões normais e de cisalhamento geradas. Os resultados mostraram que a base que melhor se adaptou à superfície vestibular construída foi a da marca Unitek, seguida pela marca A-Company, Abzil-Lancer e Morelli. As deformações sofridas pelos braquetes foram inversamente proporcionais à adaptação à superfície dentária. Os maiores picos de tensão localizaram-se nas proximidades do ponto de aplicação da força. A interface adesivo/esmalte foi mais sujeita à falha na adesão que a interface braquete/adesivo. A força de cisalhamento demonstrou ser mais provável de causar falha na adesão quando comparada à força de torção.
\end{abstract}

Palavras-chave: Base de braquete. Tensões normais. Tensões de cisalhamento. Interface braquete/esmalte, elementos finitos.

* Resumo da Dissertação de Mestrado em Odontologia - Área de Ortodontia - COP-PUC/MG

** Mestre em Ortodontia e Ortopedia Dentofacial - COP/PUC-MG.

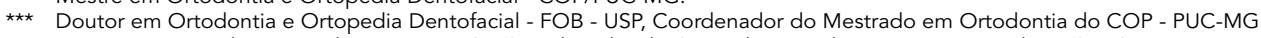

**** Doutor em Engenharia Metalúrgica - UFMG - Coordenador do Curso de Engenharia Mecatrônica da PUC-MG. 


\section{INTRODUÇÃO}

Uma grande importância tem sido dada ao desenvolvimento de braquetes ortodônticos do tipo "Straight Wire" que visam ao controle tridimensional dos dentes por meio da incorporação de dobras de $1^{\text {a }}, 2^{\text {a }}$ e $3^{\text {a }}$ ordens em sua arquitetura. Entretanto, para um adequado funcionamento desse sistema, o correto posicionamento dos acessórios sobre a superfície vestibular dos dentes torna-se imprescindivel $1^{1,2,3,4,7,10,11,14,21,22,24}$. Nesse aspecto, a similaridade entre a anatomia dentária e a curvatura da base do braquete torna-se fundamental.

Alguns autores $5,6,10,12,24,25,28,29$ observaram uma significante variação na curvatura anatômica da superfície vestibular dos dentes, o que pode comprometer o posicionamento ideal do braquete e, conseqüentemente, o funcionamento da técnica do arco contínuo.

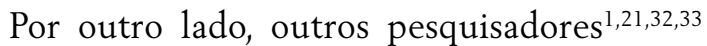
afirmam que existe uma pequena variabilidade na morfologia dentária entre os indivíduos e esta variabilidade obedece à curva normal de distribuição numa população. Conseqüentemente, as pequenas variações na forma dental não influenciam o correto posicionamento dos acessórios fabricados com os valores médios para essas curvaturas.

Em relação aos braquetes, em recente trabalho, Gontijo $^{12}$ mostrou que não existe uma padronização dos valores dos ângulos mésio-distal e ocluso-gengival das curvaturas das bases de diversas marcas. Para cada marca comercial testada, essas curvaturas apresentaram valores diferentes, o que possibilita erros de posicionamento dos acessórios, dificuldade de adaptação dos mesmos à superfície dentária e diferentes resultados aos testes de resistência à aplicação de forças.

Diante desse fato, como podemos avaliar a resistência à aplicação de forças aos braquetes se os diversos trabalhos experimentais utilizam, na sua maioria das vezes, dentes extraídos cuja anatomia dentária é variável? Como interpretar esses resul- tados se as diferentes marcas comerciais apresentam diferentes curvaturas de bases?

$\mathrm{O}$ crescente desenvolvimento tecnológico em todos os campos das ciências tem facilitado o acesso e utilização de meios antes considerados difíceis e dispendiosos tanto em tempo quanto financeiramente. Dentro da Odontologia o emprego destes recursos tem permitido o desenvolvimento de novos materiais mais resistentes, estéticos e biocompatíveis. Um exemplo desta evolução tecnológica é a utilização do método dos elementos finitos proveniente da Engenharia Mecânica.

Segundo $\mathrm{Moss}^{26}$ este método consiste em uma técnica numérica a partir da qual qualquer estrutura previamente gerada em computador é discretizada, ou dividida, em pequenos elementos bidimensionais ou tridimensionais, cada um denominado elemento finito. Nas extremidades de cada elemento finito encontram-se pontos ou nós que se conectam a pelo menos outros dois elementos finitos. Juntos, o conjunto cria o que é denominado de malha. De acordo com Gallagher ${ }^{9}$, esse método permite modelar uma estrutura analiticamente através da sua subdivisão em regiões (elementos finitos) e aplicar testes mecânicos cujos comportamentos são descritos individualmente por equações algébricas.

Os resultados obtidos a partir dessa análise devem ser interpretados por meio de uma escala de cor que representa a distribuição interna das tensões geradas sobre a estrutura. Assim, um modelo quando submetido a um determinado tipo de força produzirá tensões que sofrerão variações ao longo de toda a superfície avaliada. Essas diferentes tensões são visualizadas por cores especificas cujos valores são informados em megapascal (MPa) dentro da escala de cor.

O método dos elementos finitos tem sido utilizado por diversos autores para a avaliação da aplicação de forças sobre os braquetes durante esses últimos anos. Esse método permite eliminar diversas variáveis, controlando laboratorialmente 
os experimentos e calculando a distribuição das forças nas áreas de interface braquete/resina/den$\mathrm{te}^{15,16,17,18,19,27,30}$.

Dessa forma, a partir do método dos elementos finitos, este trabalho tem como objetivos comparar a adaptabilidade das bases dos braquetes de diferentes marcas comerciais à superfície vestibular de um dente modelado pelo método dos elementos finitos e a influência da curvatura dessas bases de braquetes nas deformações e tensões geradas durante a aplicação de forças de torção e cisalhamento.

\section{MATERIAIS E MÉTODOS}

Para realização deste trabalho foi desenvolvido um modelo matemático tridimensional de uma interface braquete/adesivo/esmalte. O dente selecionado para o estudo foi um canino inferior cujas curvaturas no sentido mésio-distal e oclusogengival foram obtidas a partir de uma média de um grupo de 30 dentes de indivíduos brasileiros adultos $^{31}$.

Para a obtenção da superfície vestibular média do canino, moldaram-se, com silicone de condensação (Silon, Dentsply-Herpo), indivíduos brasileiros que nunca haviam sido submetidos ao tratamento ortodôntico e que apresentavam a superfície vestibular hígida ao exame clínico direto. Os modelos, obtidos em gesso pedra especial Tipo IV (Herostone), foram preparados e colocados em uma base metálica desenvolvida no Departamento de Engenharia Mecânica da PUC-Minas que se adaptava perfeitamente ao recortador de gesso (Fig. 1, 2). A base metálica apresentava um dispositivo milimétrico com precisão de décimos de milímetros que permitia o desgaste da superfície incisal do dente em secções de $1 \mathrm{~mm}$ (Fig. 2). Após cada recorte, a superfície vestibular do dente era demarcada com lápis preto HB (Fig. 3) e o modelo de gesso era então posicionado em um scanner de mesa S540 (TCE) (Fig. 4), com a superfície incisal voltada para a área de scaneamento, obtendo-se a imagem da curvatura vestibular do dente. Esse processo se repetia até que toda a superfície dentária fosse desgastada e scaneada.

De posse das imagens escaneadas, utilizouse o programa de computador AutoCad2000 da Autodesk para a reconstrução dos dentes em um ambiente tridimensional, o que, posteriormente, permitiu a construção de um modelo tridimensional do canino inferior com as curvaturas médias, no sentido ocluso-gengival e mésio-distal da anatomia vestibular do canino inferior (Quadro 1).

Para a construção das bases dos braquetes no ambiente tridimensional utilizou-se as medidas encontradas em estudo prévio $^{12}$ realizado nos Departamentos de Ortodontia e de Engenharia Mecânica da PUC-Minas (Quadro 2). Dessa forma foram reconstruídas as bases dos braquetes "Straight-Wire" correspondentes ao canino inferior das marcas comerciais Morelli (modelo: 10.10.901-CRTZ-01- lote20000809, prescrição Roth-.022“ x .030”); Abzil-Lancer (modelo: Kirium Lite .022", prescrição Roth); Unitek (modelo: Gemini mesh .022", prescrição Roth ref:119701); A-Company (modelo True Straight-Wire, tamanho médio, prescrição Roth) (Fig. 5).

Os modelos em elementos finitos criados representaram a interface superfície do esmalte, camada adesiva e base de braquete correspondentes às marcas testadas. Empregou-se o programa para a análise numérica Algor com os módulos Superdraw III e SuperView, que possibilitou efetuar as etapas de pré-processamento, cálculos matemáticos e pós-processamento dos problemas.

A fim de diminuir o tamanho e a complexidade da malha gerada e também o tempo de processamento necessário, realizou-se a modelagem somente das bases dos braquetes e da superfície vestibular do canino (esmalte) correspondente à região que receberia essas bases. O restante da superfície dentária foi substituído por apropriadas condições de contorno ("boundary conditions"). 


\begin{tabular}{c|c} 
Raios & Canino inferior \\
\hline Mésio-distal & $4.13 \mathrm{~mm}$ \\
\hline Ocluso-gengival & $3.81 \mathrm{~mm}$ \\
\hline
\end{tabular}

Quadro 1 - Raios obtidos da superfície vestibular do canino inferior.

\begin{tabular}{|c|c|c|}
\hline Marca & $\begin{array}{c}\text { Valor do raio mésio- } \\
\text { distal }\end{array}$ & $\begin{array}{c}\text { Valor do raio ocluso-gen- } \\
\text { gival }\end{array}$ \\
\hline Morelli & $2.82 \mathrm{~mm}$ & Infinito \\
\hline Unitek & $3.58 \mathrm{~mm}$ & $13 \mathrm{~mm}$ \\
\hline Abzil & $1.92 \mathrm{~mm}$ & $14.5 \mathrm{~mm}$ \\
\hline A Company & $4.66 \mathrm{~mm}$ & $13 \mathrm{~mm}$ \\
\hline
\end{tabular}

Quadro 2 - Raios das bases dos braquetes para canino inferior esquerdo.

(Fonte: GONTIJO'2)

\begin{tabular}{|c|c|c|}
\hline Material & $\begin{array}{c}\text { Módulo de elasticidade (E) } \\
\text { (GPa) }\end{array}$ & $\begin{array}{c}\text { Coeficiente de Poisson } \\
\text { (v) }\end{array}$ \\
\hline Esmalte & 46.89 & 0.30 \\
\hline Adesivo & 11.721 & 0.21 \\
\hline Braquete & 210.0 & 0.30 \\
\hline
\end{tabular}

Quadro 3 - Propriedades mecânicas dos modelos. (Fonte: MIDDLETON ${ }^{23}$ )

Para a modelagem da camada de cimento, selecionou-se as características mecânicas da resina ortodôntica Concise (3M) (Quadro 3) por ser o adesivo mais comumente utilizado na Ortodontia e assumiu-se que o mesmo preenchia toda a interface esmalte/base de braquete, não havendo espaços vazios, ou seja, solução de continuidade entre a base do braquete e o esmalte.

O sistema total confeccionado com a base de braquete Morelli apresentou 11533 elementos tetraédricos interconectados por 9369 nós. O modelo gerado com a base de braquete Unitek apresentou 9739 elementos e 9374 nós. Aquele criado a partir da base de braquete Abzil apresentou 10258 elementos e 8934 nós. Por fim, o modelo obtido com a base de braquete A Company apresentou 9774 elementos e 8501 nós (Fig. 6).

Padronizou-se a utilização das mesmas espes- suras tomadas em pontos definidos. Assim, as bases dos braquetes apresentaram espessura de $0.1 \mathrm{~mm}$ e a camada adesiva uma espessura de $0.3 \mathrm{~mm}$ medida a partir do ponto central da base do braquete até a superfície do dente.

Para que o modelo pudesse ser representado de maneira simplificada e fidedigna, os materiais foram considerados homogêneos, isotrópicos e linearmente elásticos. As propriedades mecânicas empregadas foram obtidas por Middleton ${ }^{23}$ e estão descritas no quadro 3 .

Forças de $1 \mathrm{~N}(0.1 \mathrm{Kgf}=100 \mathrm{gf})$ no centro da base do braquete e também um binário de torção de $1 \mathrm{~N}$ a uma distância de $0.33 \mathrm{~mm}$ do centro da base do braquete foram aplicados ao modelo. A força foi aplicada na direção $Z$, no plano $X-Z$, enquanto o binário de torção foi aplicado no plano X-Z (Fig. 7).

As respostas às forças aplicadas ou tensões geradas foram avaliadas através da média das tensões normais (ou "von mises") e das tensões de cisalhamento (ou "tresca"), obtidas separadamente a partir da aplicação tanto da força na direção $\mathrm{Z}$ quanto do binário de torção.

\section{RESULTADOS}

Os resultados das comparações entre a curvatura da superfície vestibular do canino inferior e as curvaturas das bases dos braquetes correspondentes utilizados estão descritos na tabela 1 .

Os resultados da aplicação dos testes de resistência ao deslocamento, utilizando-se o MEF podem ser visualizados nas figuras $8,9,10$ e 11 .

\begin{tabular}{|c|c|c|}
$\begin{array}{l}\text { Tabela 1 - Comparações dos raios das } \\
\text { quetes para canino inferior. }\end{array}$ & $\begin{array}{c}\text { bases dos bra- } \\
\begin{array}{c}\text { Braquete/Curvatura do raio } \\
\text { vestibular }\end{array}\end{array}$ & $\begin{array}{c}\text { Valor do raio } \\
\text { mésio-distal }\end{array}$ \\
\hline $\begin{array}{c}\text { Curvatura vestibular } \\
\text { ocluso-gengival }\end{array}$ \\
\hline Morelli & $4.13 \mathrm{~mm}$ & $3.81 \mathrm{~mm}$ \\
\hline Unitek & $2.82 \mathrm{~mm}$ & Infinito \\
\hline Abzil & $3.58 \mathrm{~mm}$ & $13 \mathrm{~mm}$ \\
\hline A Company & $1.92 \mathrm{~mm}$ & $14.5 \mathrm{~mm}$ \\
\hline
\end{tabular}




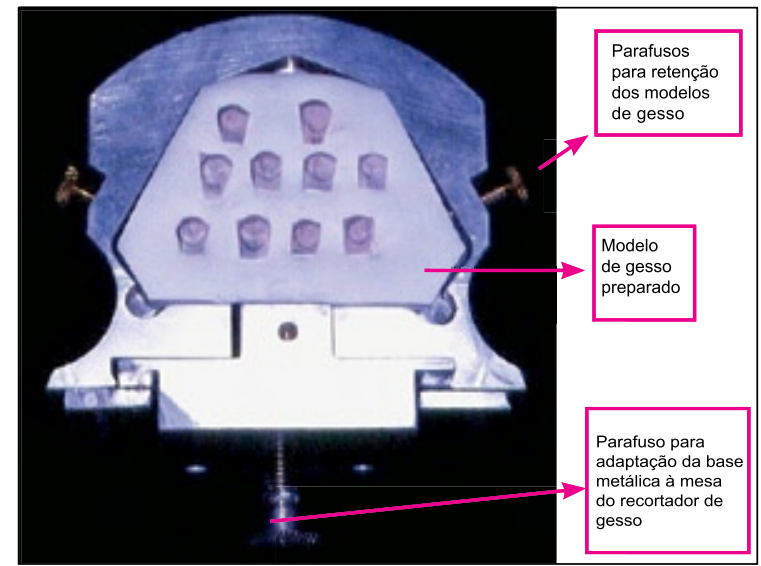

FIGURA 1 - Modelo de gesso posicionado na base metálica.

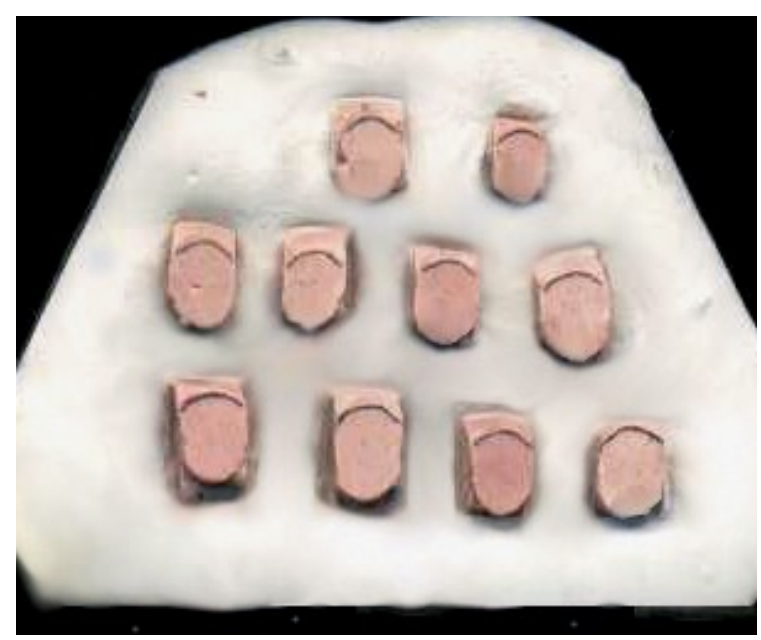

FIGURA 3 - Superfície vestibular do dente demarcada com lápis preto HB.

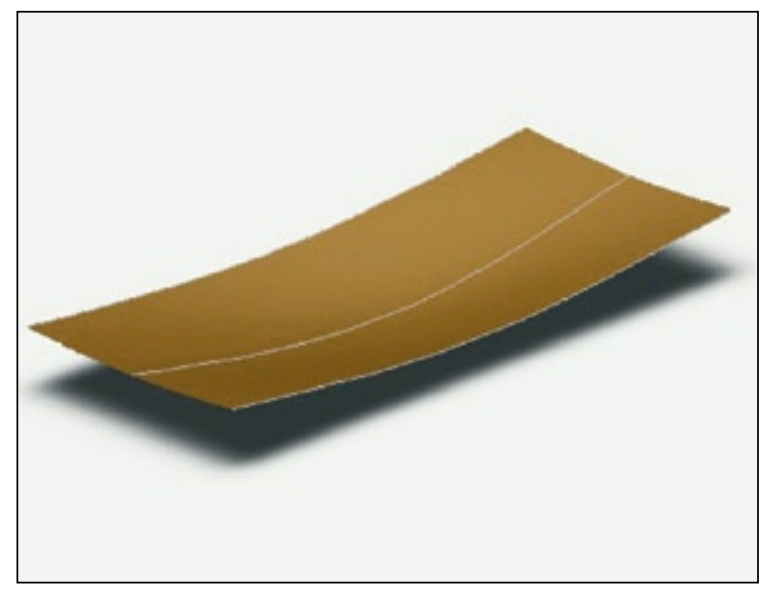

FIGURA 5 - Base de braquete Unitek em ambiente tridimensional gerada através do software AutoCAD 2000, Autodesk (Fonte: GONTIJ0 ${ }^{12}, 2002$ ).

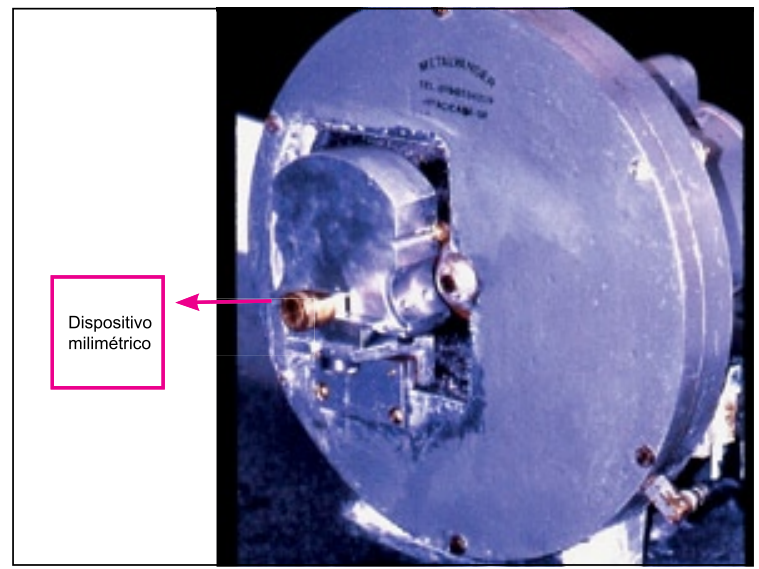

FIGURA 2 - Base metálica posicionada no recortador de gesso.

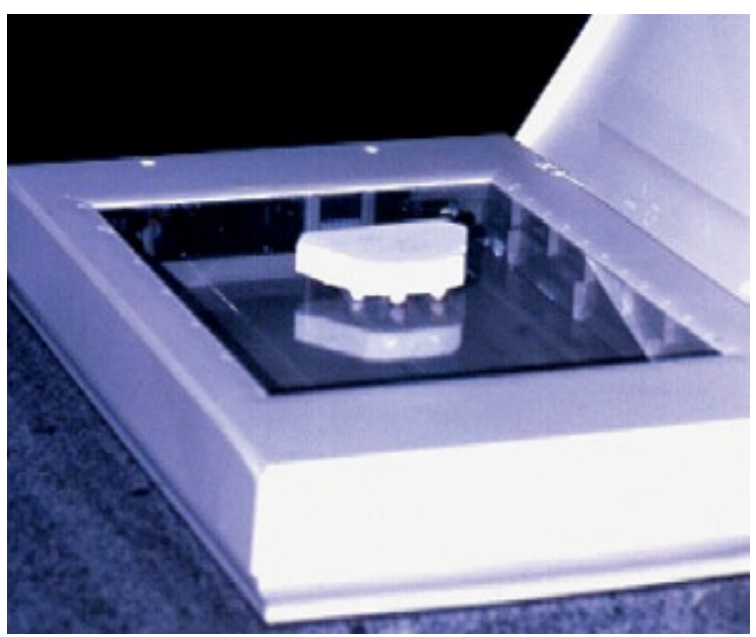

FIGURA 4 - Modelo de gesso posicionado sobre a mesa do scanner modelo S540 TCE.

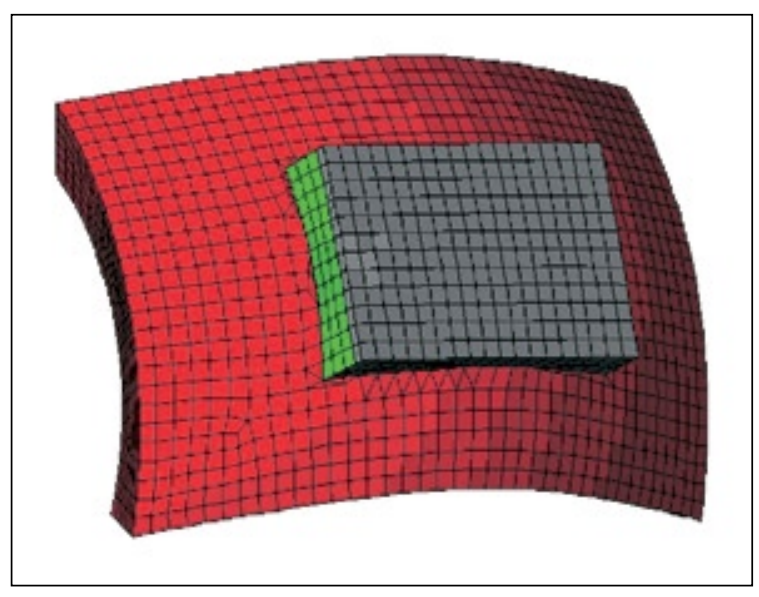

FIGURA 6 - Malha de elementos finitos do sistema base de braquete/adesivo/esmalte. As cores cinza, verde e vermelho correspondem à base do braquete, à camada adesiva e à superfície do esmalte, respectivamente. 


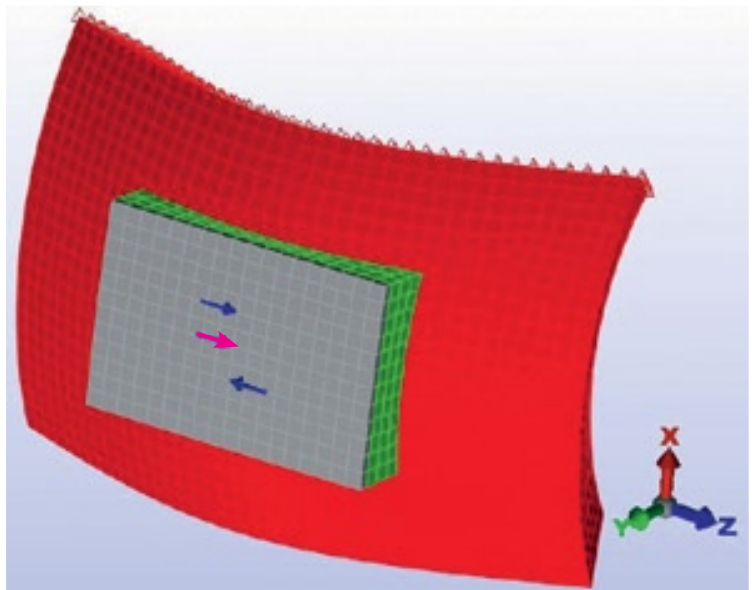

FIGURA 6 - Forças aplicadas sobre a malha de elementos finitos do sistema base de braquete/adesivo/esmalte. A seta na cor rosa corresponde à força aplicada na direção Z. As setas azuis correspondem ao binário aplicado ao plano X-Z.

\section{DISCUSSÃO}

Este trabalho buscou avaliar comparativamente, a partir de uniões ortodônticas do tipo braquete/adesivo/esmalte, a influência das curvaturas das bases dos braquetes das marcas comerciais Morelli, Unitek, Abzil-Lancer e A-Company, através das tensões geradas pela aplicação de uma força no ponto central das bases dos braquetes e de um binário promovendo torção em torno desse mesmo ponto. Para tal avaliação, utilizou-se o Método dos Elementos Finitos.

De acordo com Katona e Moore ${ }^{18}$, os trabalhos que utilizam avaliações in vitro da força de adesão do braquete ao dente estão sujeitos a variáveis como preparação da superfície dentária, tipo de adesivo, material do braquete, desenho da base do braquete, tempo de armazenamento e condições antes do teste, que tornam bastante difíceis as comparações de resultados entre dois estudos, podendo comprometer suas conclusões. Essas observações foram corroboradas por Fox, McCabe e Buckley ${ }^{8}$ após a comparação de 66 artigos relacionados a testes "in vitro" da união ortodôntica. Além disso, Katona ${ }^{17}$ lembra que a maneira pela qual os resultados dos testes realizados in vitro são descritos, ou seja, através dos valores médios obtidos dividindo-se a força aplicada pela área da base do braquete, não caracteriza adequadamente a real distribuição dessas forças, já que o método dos elementos finitos demonstrou claramente que as tensões geradas dentro da camada adesiva, da superfície do esmalte e do braquete não são uniformes. Nesse sentido, o método dos elementos finitos é vantajoso, pois permite uma boa visão da distribuição interna de forças ${ }^{19}$, além de ser capaz de representar estruturas complexas e altamente irregulares quando sujeitas a carregamentos ${ }^{9} \mathrm{e}$ proporcionar a dureza do modelo a ser estudado de maneira correta ${ }^{34}$.

A comparação entre as diversas marcas comerciais de acessórios ortodônticos confirmou os achados de Gontijo ${ }^{12}$ quanto à falta de padronização das curvaturas das bases dos braquetes. Além disso, demonstrou que nenhum braquete comercial apresentava uma geometria da base similar àquela da superfície dentária para as amostras avaliadas (Tab. 1). Aqueles braquetes cujas curvaturas de bases mais se aproximaram da medida obtida para a superfície vestibular do canino inferior foram os das marcas Unitek e ACompany. As bases das marcas Morelli e Abzil exibiram curvaturas mais discrepantes em relação à superfície dental obtida no sentido mésio-distal e, no sentido ocluso-gengival. A base Morelli, para o modelo de braquete analisado, apresentou-se plana, sem nenhum tipo de curvatura, fato este que se deve, segundo Gontijo $^{12}$, ao processo de fabricação da mesma que dificulta a incorporação de curvatura nas duas direções.

Neste trabalho, os modelos avaliados não apresentaram uma distribuição uniforme das tensões normais e de cisalhamento ao longo de suas superfícies. A base que mais se aproximou de um padrão de uniformidade foi a da marca Unitek, mas, mesmo assim, foram visíveis as variações das tensões geradas ao longo de sua 
VIANA, C. P.; MAZZIEIRO, E. T.; LANDRE JR., J..
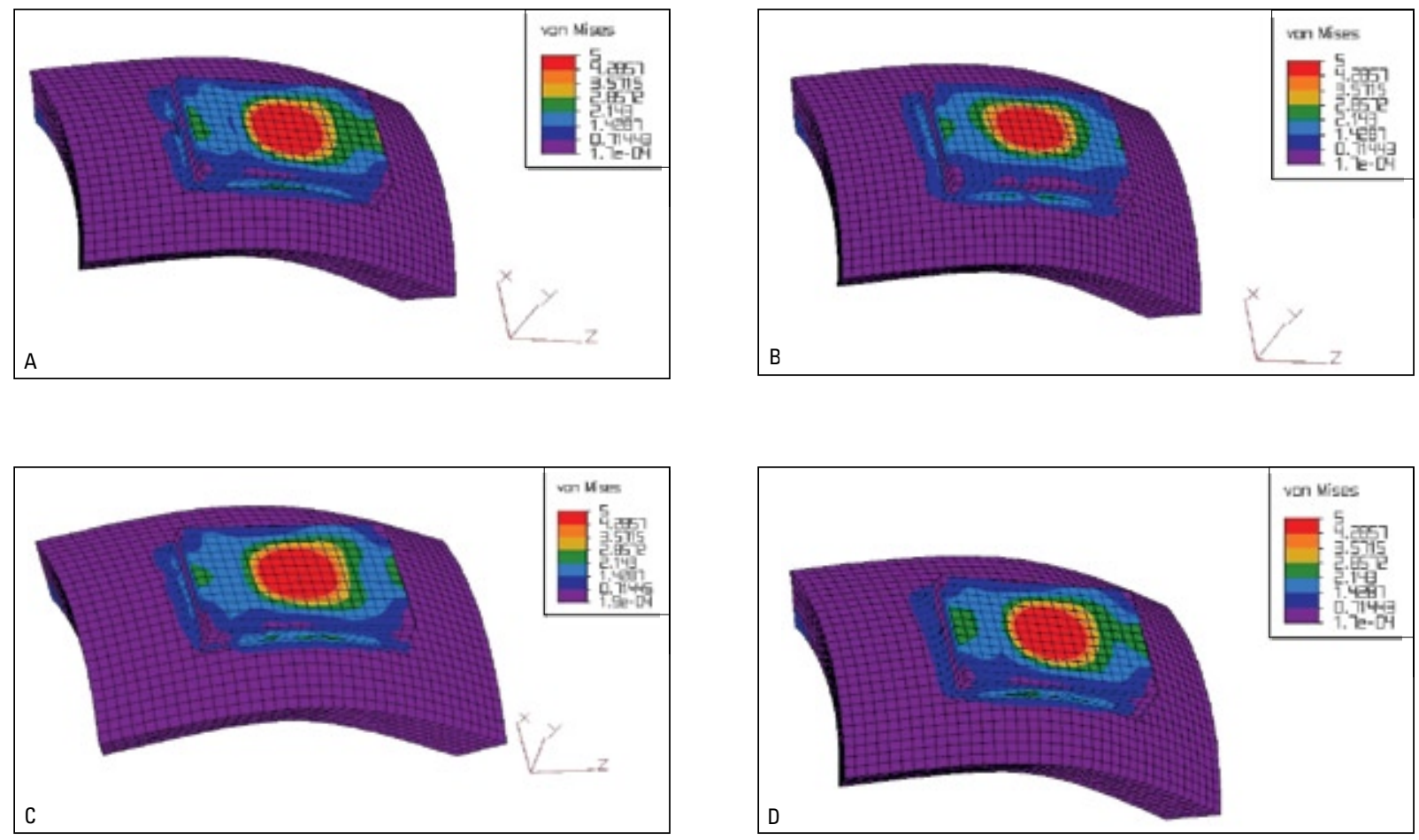

FIGURA 8 - Tensões normais geradas pela força na direção Z. A) Morelli. B) Unitek. C) Abzil-Lancer. D) A-Company.
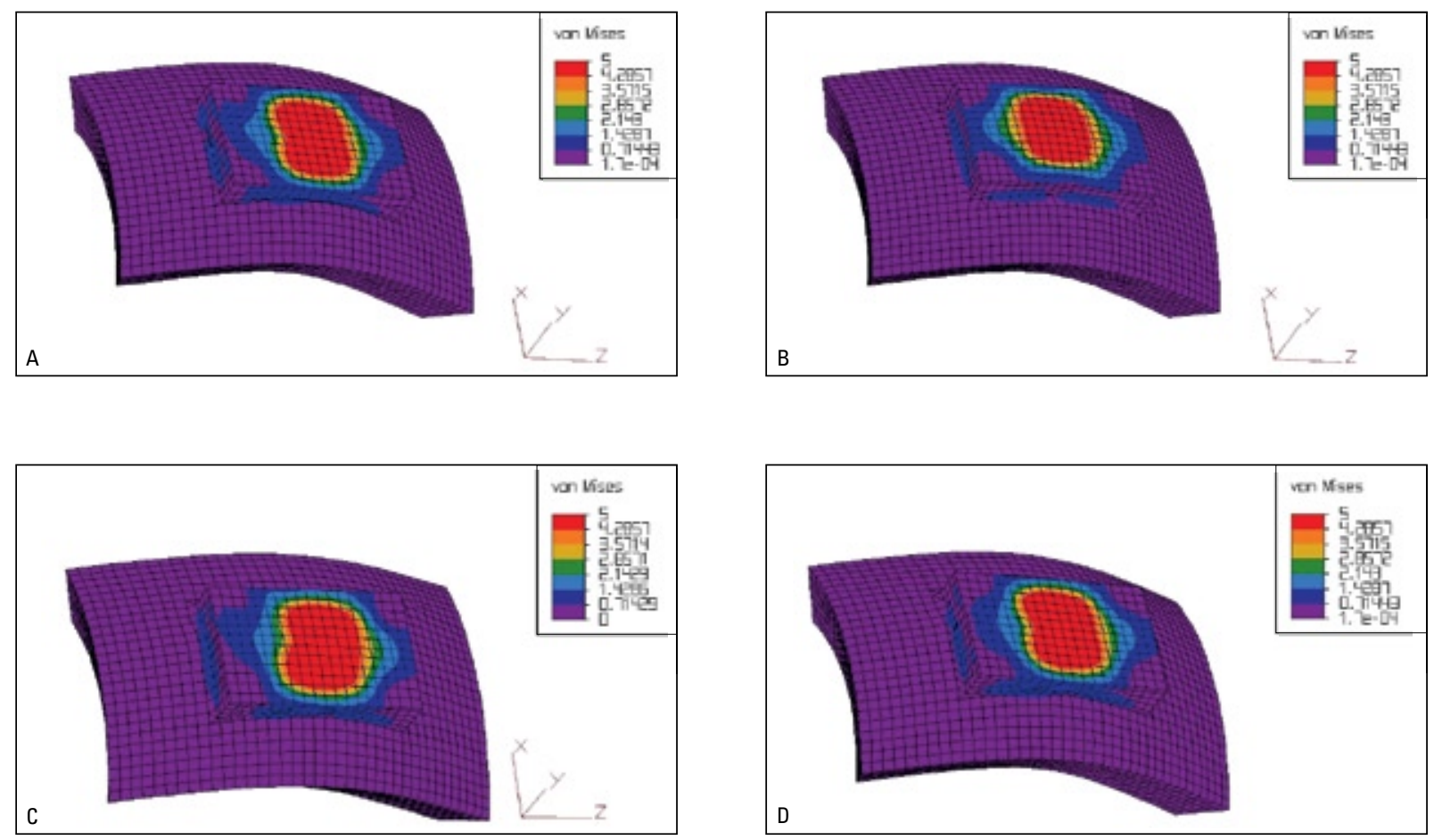

FIGURA 9 - Tensões normais geradas pelo binário. A) Morelli. B) Unitek. C) Abzil-Lancer. D) A-Company. 

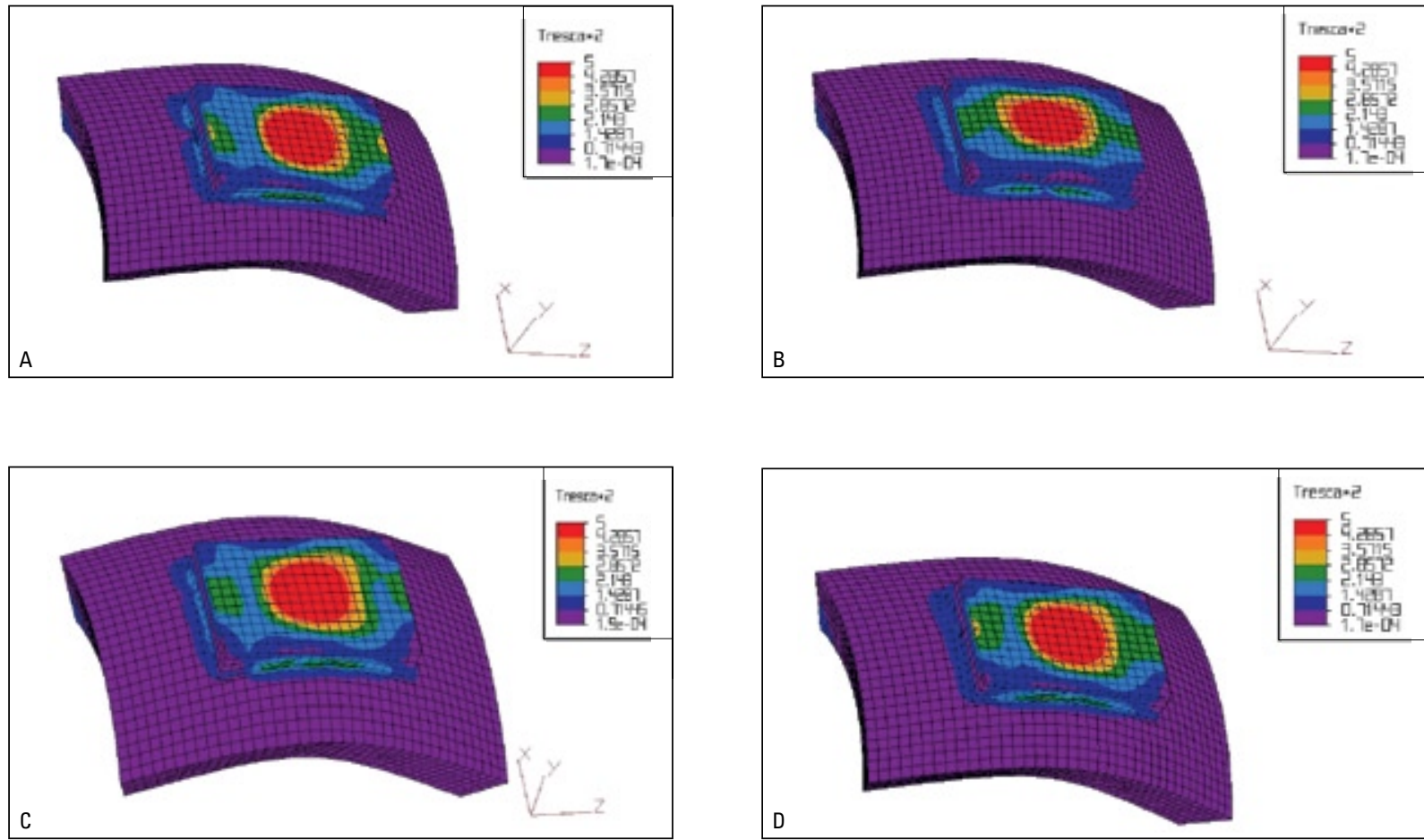

FIGURA 10 - Tensões de cisalhamento geradas pela força na direção Z. A) Morelli. B) Unitek. C) Abzil-Lancer. D) A-Company.
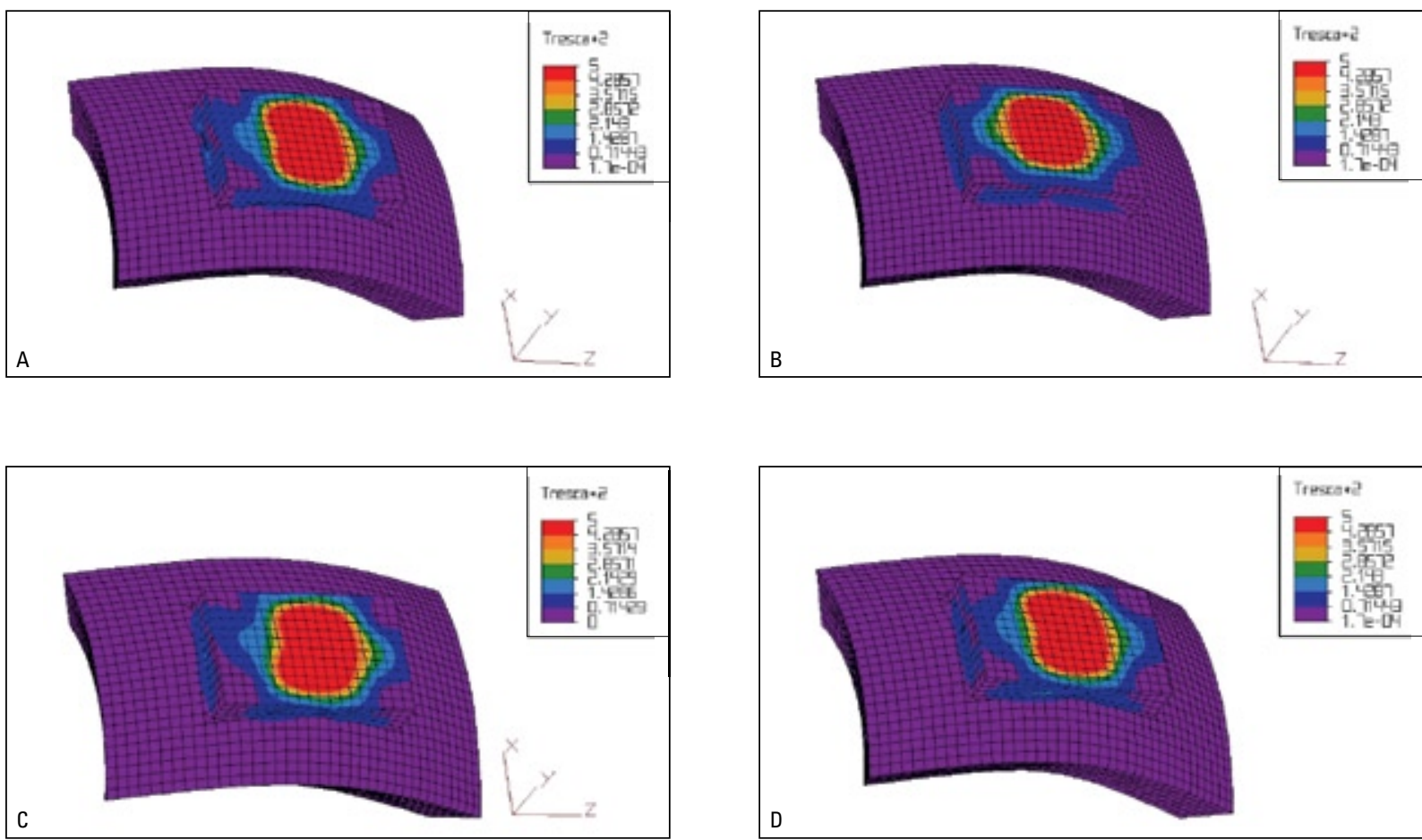

FIGURA 11 - Tensões de cisalhamento geradas pelo binário. A) Morelli. B) Unitek. C) Abzil-Lancer. D) A-Company 
extensão e na camada adesiva. Esses resultados também foram observados por Katona ${ }^{15}$ e Katona e Moore ${ }^{18}$ que, apesar de utilizarem um modelo bidimensional, mostraram uma distribuição não uniforme das tensões dentro da camada de cimento de uma união ortodôntica. Katona $^{17}$, Thomas, Rijk e Evans ${ }^{30}$ e Knox ${ }^{20}$ observaram essa mesma ausência de uniformidade baseando-se em modelos tridimensionais. Essa coincidência de resultados pode ser explicada, de acordo com Katona e Moore ${ }^{18}$, pelas curvaturas e irregularidades da superfície do esmalte e pelo efeito das tensões nas extremidades da camada adesiva, que ocorrem mesmo quando se considera a camada de cimento plana e lisa.

Apesar dessa falta de uniformidade na distribuição das tensões, este estudo mostrou, da mesma maneira que Katona ${ }^{17}$, que as maiores concentrações de esforços localizavam-se nas proximidades do local de aplicação da força e diminuíam à medida que se distanciava desse ponto.

As tensões normais ("von mises") e tensões de cisalhamento geradas pela aplicação da força e do binário demonstraram que as regiões mais sujeitas à falha de adesão corresponderiam àquelas da interface adesivo/esmalte. Observação semelhante foi feita por Katona ${ }^{15}$ a partir de variações na angulação da aplicação da força, na qual também verificou que o local mais provável para ocorrência da falha na adesão seria na interface cimento/esmalte.

Avaliando a interface esmalte/adesivo, as tensões normais e de cisalhamento produzidas pela aplicação da força na direção $Z$ (cisalhamento) (Fig. 8 e 10) foram maiores (até 2.8572 $\mathrm{MPa}$ ) que as geradas pela aplicação do binário de torção (até 1.4287 MPa) (Fig. 9, 11), o que sugere uma falha na adesão nessa interface principalmente quando sujeita a forças que geram cisalhamento, fato este também observado por Katona $^{16}$. A base de braquete Unitek demonstrou campos de tensões menores e mais uniformes quando comparada com as demais marcas
(Fig. 8B, 9B, 10B, 11B). Esse fato se justifica pela melhor adaptabilidade da base à superfície dentária modelada.

Para Katona ${ }^{17}$, as tensões, tanto na interface braquete/cimento quanto na interface cimento/ esmalte, apresentaram-se similares quando submetidas a forças de torção. Este estudo demonstrou que, apesar das tensões geradas pela aplicação do binário de torção serem menores que aquelas produzidas pela força de cisalhamento, existe uma maior tendência para ruptura da adesão na interface esmalte/adesivo também com a aplicação desse binário de torção, principalmente para as bases das marcas Morelli, Abzil-Lancer e A-Company (Fig. 9, 11).

$\mathrm{Knox}^{19}$ observou que as forças de cisalhamento geram maiores tensões nas proximidades das margens do braquete, cimento e esmalte. Um resultado semelhante pode ser visto neste trabalho, já que todas as bases avaliadas exibiram concentrações de tensões nas laterais quando sujeitas à força na direção Z (Fig. 8, 10). Mais uma vez a base Unitek apresentou tensões distribuídas mais uniformemente que as bases Morelli, Abzil-Lancer e A-Company.

Portanto, baseando-se nos resultados deste estudo, a base de braquete que melhor se portou nos diversos testes aplicados através do método dos elementos finitos foi a da marca Unitek, seguida pelas bases A-Company, Abzil-Lancer e Morelli. Em relação à curvatura de base propriamente dita, observou-se que os resultados dos testes aplicados foram diretamente proporcionais a suas curvaturas, o que sugere uma relação direta entre a curvatura da base do braquete, adaptação à superfície vestibular dos dentes e resistência à aplicação de forças.

Todos esses aspectos aqui descritos devem ser levados em consideração durante a escolha de um braquete para sua utilização na clínica, principalmente naqueles casos nos quais os dentes apresentem contornos mais acentuados e formas anatômicas mais irregulares, já que bases 
com curvaturas mésio-distais similares àquelas dos dentes e também a incorporação de curvaturas no sentido ocluso-gengival favorecem uma melhor adaptação dessas bases sobre a superfície do esmalte, o que gera deformações menores e mais uniformes, além de campos de tensões distribuídos de maneira mais simétrica no centro e ao longo de toda a extensão da base do braquete.

Clinicamente é importante ressaltar que os resultados são significativos em relação à necessidade de curvaturas de bases de braquetes mais adaptadas à anatomia dentária, porém devemos lembrar que esse trabalho avaliou somente a influência da curvatura das bases dos braquetes, ou seja, a adaptação destas bases a uma superfície dentária cujo contorno foi estabelecido pela média dos contornos vestibulares de 30 dentes. Maiores avaliações se fazem necessárias para o estabelecimento de padrões médios de curvaturas dentárias, determinando-se as angulações mésio-distais e ocluso-gengivais "ideais" para a curvatura de cada base de braquete.

Além disso, outras variáveis que regem a adesão do braquete ao dente, como as variações na anatomia dental entre os indivíduos, a variedade de resinas disponíveis e os diferentes tipos de malhas das bases dos braquetes podem, para um paciente em particular, tornar superior os resultados de uma base de braquete que se portou de maneira inferior nesse trabalho.

Assim, apesar desse estudo sugerir uma superioridade da curvatura da base de braquete da marca Unitek em relação às demais marcas analisadas, o mesmo pode não se adaptar bem em um paciente cuja curvatura vestibular dos dentes seja muito diferente da curvatura da base do braquete Unitek. Além disso, a utilização das modernas resinas disponíveis no mercado pode também melhorar a resistência de uniões ortodônticas que utilizam braquetes cujas bases apresentam um contorno menos eficiente. Finalmente, as várias espessuras e configurações das malhas das bases dos braquetes podem aumentar ou diminuir a capacidade adesiva do braquete ao dente.

\section{CONCLUSÕES}

A partir das observações e dos resultados obtidos da comparação e dos testes aplicados utilizando-se o método dos elementos finitos podese concluir que:

1) Em relação à adaptabilidade das bases dos braquetes, as curvaturas que mais aproximaram das curvaturas obtidas para a superfície vestibular do canino inferior modelado foram as da marca Unitek, seguida pela A-Company, Abzil-Lancer e Morelli;

2) Em relação à influência da curvatura dessas bases de braquetes nas deformações e tensões geradas durante a aplicação de forças de torção e cisalhamento concluiu-se que:

- Quanto maior a adaptação da base do braquete ao dente, mais uniforme será a distribuição das forças ao longo de sua superfície;

- As maiores concentrações de tensões nos modelos localizaram-se no ponto de aplicação da força e diminuíram à medida que se distanciavam deste ponto;

- As tensões geradas demonstraram que as regiões mais sujeitas à falha de adesão corresponderiam àquelas da interface adesivo/esmalte para todas as marcas testadas;

- Para todas as bases avaliadas, a força de cisalhamento demonstrou ser mais provável de causar falha na adesão quando comparada à força de torção. 


\title{
The influence of the variation of the bracket base curvature in a bonded orthodontic attachment submitted by different load cases using the finite element method
}

\begin{abstract}
The objective of this study was to evaluate the adaptability of the four straight-wire brackets bases (Morelli, Unitek, A-Company and Abzil-Lancer) to facial surface of a lower canine. The lower canine facial curvature data to be used in the three-dimensional finite element model were established by 30 Brazilian adult individuals and the brackets bases curvature used were described in a prior study. The three-dimensional finite element analysis of the interface enamel/cement/base of bracket for each tested mark was used. Loads of $1 \mathrm{~N}$ promoting lateral displacement and torsion had been applied in the center of the base of brackets and the distribution of these efforts on the models was observed as well as the normal and shear tensions generated. The results had shown that the base that better fit to facial surface was the one from Unitek, followed by the A-Company, Abzil-Lancer and Morelli. The brackets deformations were inversely proportional to its adaptation. The biggest peaks of tensions had been situated near to the point of force application. The cement/enamel interface was more likely to fail in the adhesion that the bracket/cement interface. The torsion load was less likely to cause damage in the adhesion when compared with the shear load.
\end{abstract}

Key words: Bracket base. Normal tensions. Shear tensions. Bracket/enamel interface. Finite element.

\section{REFERÊNCIAS}

1. ANDREWS, L. F. Straight-wire appliance: origin, controversy, commentary. J Clin Orthod, Boulder, v. 10, no.2, p. 99-114, Feb. 1976.

2. ANDREWS, L. F. Straight-wire appliance: explained and compared. J Clin Orthod, Boulder, v. 10, no. 3, p. 174-194, Mar. 1976.

3. ANDREWS, L. F. Straight-wire appliance: arch form, wire bending \& an experiment. J Clin Orthod, Boulder, v. 10, no. 8, p. 581-588, Aug. 1976.

4. BALUT, N.; KLAPPER, L.; SANDRIK, J.; BOWMAN, D. Variations in bracket placement in preajusted orthodontic appliance. Am J Orthod Dentofacial Orthop, St. Louis, v. 102, no. 1, p. 62-67, July 1992.

5. BRYANT, R. M.; SADOWSKY, P. L.; HAZEIRIG, J. B. Variability in three morphologic features of the permanent maxillary central incisor. Am J Orthod, St. Louis, v. 86, no. 1, p. 25-32, July 1984.

6. CARLSSON, R.; RÖNNERMAN, A. Crown-root angles of upper central incisors. Am J Orthod, St. Louis, v. 64, no. 2, p.147-154, Aug. 1973.

7. DELLINGER, E. L. A scientific assessment of the straight-wire appliance. Am J Orthod, St. Louis, v. 73, no. 2, p. 290-299, Mar. 1978.

8. FOX, N. G.; MCCABE, J. F.; BUCKLEY, J. G. A critique of bond strength testing in orthodontics. Br J Orthod, London, v. 21, no.1, p. 33-43, Feb. 1994.

9. GALLAGHER, R. H. Finite element analysis: fundamentals. Englewood Cliffs: Prentice-Hall, 1975.
10. GERMANE, N.; BENTLEY, B. E.; ISAACSON, R. J. Three biologic variables modifying faciolingual tooth angulation by straight-wire appliances. Am J Orthod, St. Louis, v. 96, no. 4, p. 312-319, Oct. 1989.

11. GERMANE, N.; BENTLEY, B.; ISAACSON, R. J.; REVERE JR., $\mathrm{J}$. $\mathrm{H}$. The morphology of canines in relation to preadjusted appliances. Angle Orthod, Appleton, v. 60, no. 1, p. 49-54, 1990.

12. GONTIJO, L. de P. Variação angular da curvatura, composição química e resistência da base de bráquete "straight wire". 2002. 83 f. Dissertação (Mestrado em Odontologia)-Pontifícia Universidade Católica de Minas Gerais, Belo Horizonte, 2002

13. GOOSE, D. H. Variability of the form of maxillary permanent incisors. J Dent Res, Chicago, v. 35, no. 6, p. 902-908, Dec. 1956.

14. JANSON, G.; VASCONCELOS, M. H.; BOMBONATTI, R.; FREITAS, M. R.; HENRIQUES, J. F. C. Considerações clínicas sobre o posicionamento vertical dos acessórios. R Dental Press Ortodon Ortop Facial, Maringá, v. 5, n. 3, p. 45-51, maio/jun. 2000.

15. KATONA, T. R. The effects of load location and misalignment on shear/peel treating of direct bonded orthodontic brackets: a finite element model. Am J Orthod Dentofacial Orthop, St. Louis, v. 106, no. 4, p. 395-402, Oct. 1994.

16. KATONA, T. R. Stresses developed during clinical debonding of stainless steel orthodontic brackets. Angle Orthod, Appleton, v. 67, no. 1, p. 39-46, 1997.

17. KATONA, T. R. Comparison of the stresses developed in tension, shear peel, and torsion strength testing of direct bonded orthodontic. Am J Orthod Dentofacial Orthop, St. Louis, v. 112 , no. 3, p. 244-251, Sept. 1997.

18. KATONA, T. R.; MOORE, B. K. The effects of load misalignment on tensile load testing of direct bonded orthodontic brackets - a finite element model. Am J Orthod Dentofacial Orthop, St. Louis, v.105, no. 6, p. 543-551, June 1994. 
19. KNOX, J.; JONES, M. L: HÜBSCH, P. MIDDLETON, J.; KRALJ, B. An evaluation of the stresses generated in a bonded orthodontic attachment by three different load cases using the finite element method of stress analysis. J Orthod, London, v. 27, p. 39-46, Mar. 2000.

20. KNOX, J.; KRALJ, B.; HÜBSCH, P.; MIDDLETON, J.; JONES, M. L. An evaluation of the influence of orthodontic adhesive on the stresses generated in a bonded bracket finite element model. Am J Orthod Dentofacial Orthop, St. Louis, v.119, no.1, p. 43-53, Jan. 2001

21. MAGNESS, W. B. The straight-wire concept. Am J Orthod, St. Louis, v. 73, no. 5, p. 541-550, May 1978.

22. MEYER, M.; NELSON, G. Preadjusted edgewise appliances: theory and practice. Am J Orthod, St. Louis, v.73, no. 5, p. 485-498, May 1978.

23. MIDDLETON, J., HÜBSCH, P., KRALJ, B., KNOX, J., JONES, M. L. Numerical investigation of fixed orthodontic brackets. Proc Instn Mech Eng, London, v. 214, part H, no. 3, p. 311-323, 2000.

24. MIETHKE, R. R.; MELSEN, B. Effect of variation in tooth morphology and bracket position on first and third order correction with preadjusted appliances. Am J Orthod Dentofacial Orthop, St. Louis, v. 116, no. 3, p. 329-335, Sept. 1999

25. MORROW, J. B. The angular variability of the facial surfaces of the human dentition: an evaluation of the morphological assumptions implicit in the various "straight wire" techniques. 1978. 94 f. Tese (Doutorado em Ciência da Odontologia)Universidade de Saint Louis, St. Louis, 1978.
26. MOSS, M. L. et al. Finite element method modeling of craniofacial growth. Am J Orthod, St. Louis, v. 87, no. 6, p. 453-472, June 1985.

27. ROSSOUW, P. E.; TERBLANCHE, E. Use of finite element analysis in assessing stress distribution during debonding. J Clin Orthod, Boulder, v. 29, no.11, p. 713-717, 1995.

28. TAYLOR, R. M. S. Variation in form of human teeth: I. An anthropologic and forensic study of maxillary incisors. J Dent Res, Chicago, v. 48, no. 1, p. 5-15, Feb. 1969.

29. TAYLOR, R. M. S. Variation in form of human teeth: II. An anthropologic and forensic study of maxillary canines. J Dent Res, Chicago, v. 48, no. 2, p.173-182, Mar./Apr. 1969.

30. THOMAS, R. L.; RIJK, W. G.; EVANS, C. A. Tensile and shear stresses in the orthodontic attachment adhesive layer with 3D finite element analysis. Am J Orthod Dentofacial Orthop, St. Louis, v. 116, no. 5, p. 530-532, Nov. 1999.

31. VIANA, C. P. A influência da variação da curvatura da base do bráquete numa união ortodôntica submetida a diferentes cargas através do método dos elementos finitos. 2003. $102 \mathrm{f}$ Dissertação (Mestrado em Odontologia)-Pontifícia Universidade Católica de Minas Gerais, Belo Horizonte, 2003.

32. WATANABE, K.; KOGA, M. A Morphometric study with setup models for bracket design. Angle Orthod, Appleton, v. 71, no. 6, p. 499-511, 2001.

33. WHEELER, R. C. Dental anatomy, physiology and occlusion 2nd ed. Philadelphia: W. B. Saunders, 1974.

34. YETRAM, A. L.; WRIGHT, K. W. J.; PICKARD, H. M. Finite element stress analysis of the crowns of normal and restored teeth. J Dent Res, Chicago, v. 55, no.6, p.1004-1011, Nov./Dec. 1976.
Endereço para correspondência

Cláudio Pereira Viana

COP/PUC-MG

Av. Dom José Gaspar, 500, prédio 46, Coração Eucarístico

Belo Horizonte - MG

CEP: 30535-610

E-mail: cop@pucminas.br, mazzieiro@pucminas.br 